\title{
Understanding What is Observed When Studying Complex Formulations of Consumer Products by Cryo FESEM
}

\author{
Rodman T. Heu
}

Measurement Sciences and Innovation Group, Colgate-Palmolive, Co., Piscataway Technical Center, Piscataway, New Jersey, 08855, USA

The complex nature of consumer products does not easily lend itself to study by conventional microscopy techniques. Consumer products can be readily studied by traditional optical microscopy, but these technique(s) have inherent limitations.

Conventional scanning electron microscopy (SEM) provides the required resolution and depth of field but cannot accommodate the vacuum sensitive components that are typically found in consumer products.

Consumer products can include vacuum sensitive materials such as water, surfactantwater mixtures, silicones, alcohols, fragrance, and encapsulated volatiles to name a few. Cryo-stabilization through the use of liquid nitrogen (LN) can stabilize these formulations to withstand the harsh vacuum environment that is typically required by a field emission scanning electron microscope (FESEM).

This talk will focus on the application of the technique of Cryo FESEM to the study of consumer products. Technique procedure will be reviewed in a cursory manner.

Topics of discussion will cover a wide range of personal care products and their ingredients. Specific examples of Cryo FESEM will cover the secondary electron imaging of water rich surfactant systems such as liquid detergents. Figures $1 \mathrm{a}$ and $1 \mathrm{~b}$ demonstrate what is observed on the fracture face of the sample, after in-vacuo fracturing, on a simple nonionic/anionic surfactant system as salt content varies. Basic product characterization such as oil-rich liquid soaps (body washes, shampoos) will be presented and the rationale for how image interpretation is rendered. An example of a complex liquid soap typically encountered is seen in Figures $2 a$ and $2 b$. In these images distinctly different phases are observed and related to their major ingredients. The characterization of stearyl alcohol rich personal care products also will be reviewed with a focus on the affect of cooling on structural morphology.

In addition to the aforementioned topics, the pitfalls associated with sample preparation, sample cooling, component malfunction, and experimental artifacts will be discussed [1]. Examples of how these operational anomalies can affect imaging will be presented. Additional influences upon imaging under cryo conditions due to the FESEM will also be reviewed.

\section{References}

[1] P. Echlin, Low-Temperature Microscopy and Analysis, Plenum Press, New York, 1992. 


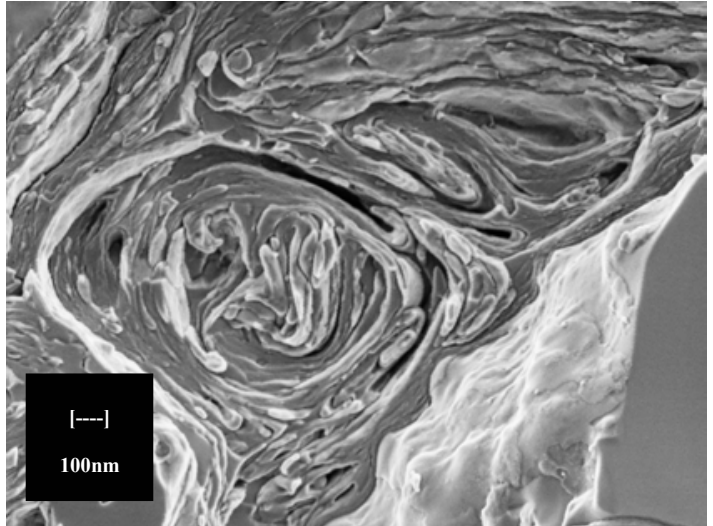

FIG. 1a.

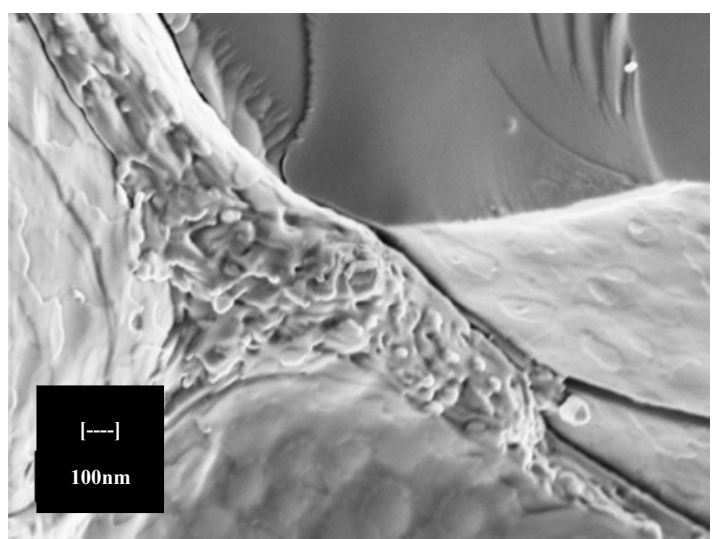

FIG. 1b.

Nonionic and anionic surfactants in aqueous base at 5\% salt, FIG. 1a, and $0 \%$ salt, FIG $1 b$.

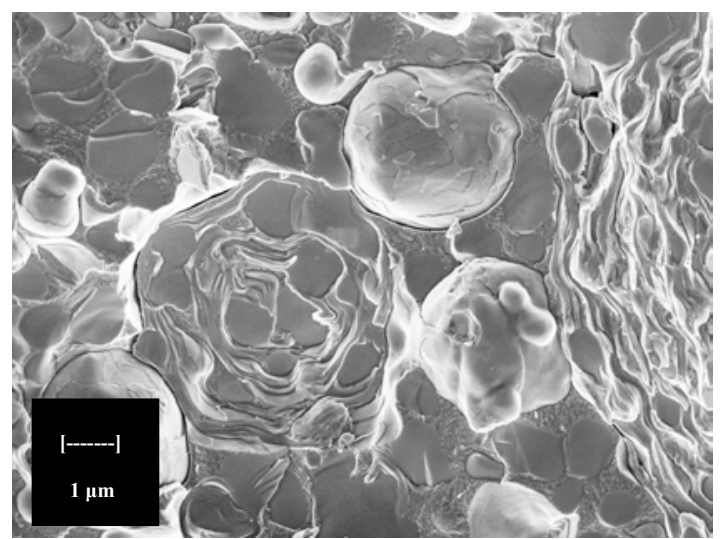

FIG. 2a.

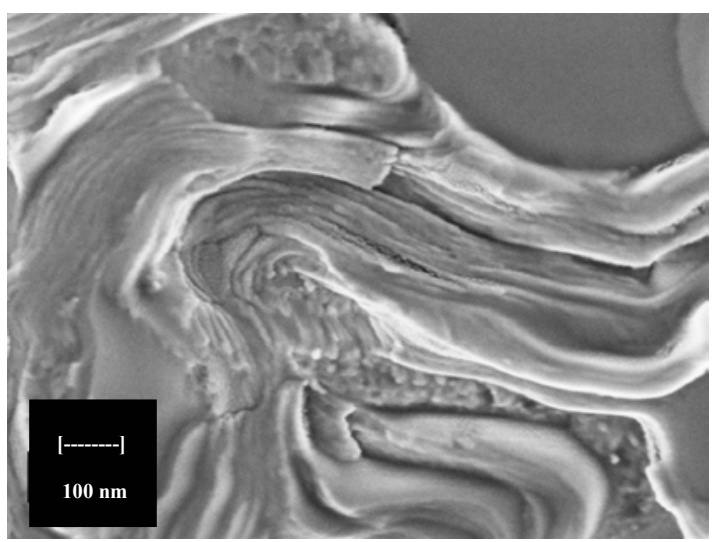

FIG. $2 b$.

Aqueous base soap containing amphoteric, anionic and nonionic surfactants. FIG. 2a reveals significantly unique structures. FIG. 2a confirms the presence of multilamellar structures. 